\title{
Natural NMSSM with a light Singlet Higgs and Singlino LSP
}

\author{
C. T. Potter ${ }^{\mathrm{a}}$ \\ Physics Department, University of Oregon, Eugene, USA
}

Received: 2 October 2015 / Accepted: 22 December 2015 / Published online: 25 January 2016

(C) The Author(s) 2016. This article is published with open access at Springerlink.com

\begin{abstract}
Supersymmetry (SUSY) is an attractive extension of the Standard Model (SM) of particle physics which solves the SM hierarchy problem. Motivated by the theoretical $\mu$-term problem of the Minimal Supersymmetric Model (MSSM), the Next-to MSSM (NMSSM) can also account for experimental deviations from the SM like the anomalous muon magnetic moment and the dark matter relic density. Natural SUSY, motivated by naturalness considerations, exhibits small fine tuning and a characteristic phenomenology with light higgsinos, stops, and gluinos. We describe a scan in NMSSM parameter space motivated by Natural SUSY and guided by the phenomenology of an NMSSM with a slightly broken Peccei-Quinn symmetry and a lightly coupled singlet. We identify a scenario which survives experimental constraints with a light singlet Higgs and a singlino lightest SUSY particle. We then discuss how the scenario is not presently excluded by searches at the Large Hadron Collider (LHC) and which channels are promising for discovery at the LHC and International Linear Collider.
\end{abstract}

\section{Introduction}

With the discovery of the $125 \mathrm{GeV}$ Higgs boson $h_{125}$ by ATLAS [1] and CMS [2] at the Large Hadron Collider (LHC), particle physics enters a new era. In the Standard Model (SM) of particle physics, the properties of the Higgs boson are determined by theory once the mass is known [3]. At present, their measurements are consistent with the SM prediction [49].

But the SM is not complete. Experimentally, it does not account for Dark Matter (DM), the anomalous muon magnetic moment or the strong CP problem, among other things. Theoretically, it suffers from the hierarchy problem. Supersymmetry (SUSY) solves the hierarchy problem by introducing a fermionic partner for each SM boson and a bosonic

\footnotetext{
a e-mail: c.t.potter@gmail.com
}

partner for each SM fermion [10]. SUSY with conserved $R$ parity provides a natural candidate for DM, the Lightest Supersymmetric Partner (LSP), and it can account for the anomalous muon magnetic moment by introducing new particles in loops.

The principle of naturalness in physics maintains that an effective physical theory approximately valid below some characteristic scale should not be very sensitive to the correct theory above that scale [11]. Applied to electroweak symmetry breaking in SUSY, this implies that the success of the effective SM Higgs theory disallows SUSY too far above the electroweak scale [12]. In particular, the characteristic mass spectrum of Natural SUSY includes light superpartners of the Higgs bosons, top quark and gluon near the electroweak scale.

The Minimal SUSY Model (MSSM) contains only the SM particles and their superpartners, together with an enlarged Higgs sector: one neutral pseudoscalar, two neutral scalars and two charged scalars which arise from the two Higgs doublets $\hat{H}_{u}$ and $\hat{H}_{d}$ necessary for the Higgs mechanism in SUSY [3]. But the MSSM suffers from the so-called $\mu$-term problem, which prevents the term $\mu \hat{H}_{u} \hat{H}_{d}$ in the MSSM superpotential from reaching the electroweak scale without fine tuning $[10,13,14]$.

The Next-to MSSM (NMSSM) solves the $\mu$-term problem by introducing a singlet $\hat{S}$ and replacing $\mu \hat{H}_{u} \hat{H}_{d}$ with $\lambda \hat{S} \hat{H}_{u} \hat{H}_{d}$. The $Z_{3}$ invariant NMSSM superpotential is $[13,14]$

$W=\lambda \hat{S} \hat{H}_{u} \hat{H}_{d}+\frac{\kappa}{3} \hat{S}^{3}$

where $\lambda$ and $\kappa$ are free parameters. An effective $\mu$-term is generated as the vacuum expectation value of $\hat{S}, \mu_{\mathrm{eff}}=\lambda\langle\hat{S}\rangle$, reaching a natural scale without fine tuning $[13,14]$.

In addition to the Higgs content of the MSSM, the NMSSM contains an additional pseudoscalar and an additional scalar so that the NMSSM Higgs sector consists of two neutral pseudoscalars $\left(a_{1}, a_{2}\right)$, three neutral scalars $\left(h_{1}, h_{2}, h_{3}\right)$ and two charged scalars $\left(H^{+}, H^{-}\right)[15,16]$. The 
NMSSM Higgs sector is fully determined at tree level by $\lambda$ and $\kappa, A_{\lambda}$, and $A_{\kappa}$ (soft trilinear couplings), $\mu_{\text {eff }}$ and $\tan \beta$ (ratio of $H_{u}, H_{d}$ vacuum expectation values) [15].

One notable version of the NMSSM is the Peccei-Quinn (PQ) symmetric NMSSM, characterized by $\kappa=0$ [13-15, 17]. The PQ symmetric NMSSM explains why there is so little $\mathrm{CP}$ violation in the strong sector by exhibiting an axion, the massless pseudoscalar $a_{1}$. In the NMSSM with a slightly broken PQ symmetry, with small $\kappa$ and $A_{\kappa}$, the $a_{1}$ acquires a small mass proportional to $\kappa A_{\kappa}$ but can still solve the strong CP problem [15, 18-21].

Scenarios with a light NMSSM pseudoscalar Higgs, motivated variously by the strong $\mathrm{CP}$ problem, naturalness, the anomalous muon magnetic moment, the $\eta_{b}$ mass spectrum, and the similarity of the baryon density to the dark matter density, have been discussed in the literature [15,22-28]. In this study we assume a light NMSSM pseudoscalar $a_{1}$ with $2 m_{\tau}<m_{a_{1}}<2 m_{B}$. Motivated by the LEP $Z b \bar{b}$ feature near $m_{b \bar{b}} \approx 60 \mathrm{GeV}$ [29], we identify this as an $h_{1}$ candidate. We further identify the $h_{125}$ as the second lightest neutral scalar $h_{2}$ of the NMSSM and note that the $h_{125}$ signal strength measurements at the LHC [5,6] place the heavier NMSSM $a_{2}, h_{3}, H^{+}$in the effective MSSM decoupling limit.

\section{Effective $\operatorname{MSSM}(\lambda, \kappa \approx 0)$}

We now consider the phenomenology of the NMSSM with a slightly broken PQ symmetry in which the singlet $S$ is completely decoupled from the doublets $H_{u}$ and $H_{d}(\lambda=0)$. We then consider how the phenomenology is altered when the singlet is allowed a weak coupling to the doublets $(\lambda \approx 0)$. The case $\lambda, \kappa \approx 0$ is known as the effective MSSM [14].

For $\lambda=0$, there is no mixing of the singlet with the doublets. The generic couplings in the NMSSM have been detailed in $[14,30]$. We adopt the notation of the former, denoting $S_{i j}^{2}\left(P_{i j}^{2}\right)$ as the $j$ th component of mass eigenstate $h_{i}\left(a_{i}\right)$, where $j=1,2,3$ corresponds to the $u$ doublet, the $d$ doublet, and singlet respectively. For purely singlet $h_{1}$ and $a_{1}, S_{13}=P_{13}=1$, and all other $S_{1 j}, P_{1 j}$ vanish, so the $a_{1}$ cannot decay to SM particles since their coupling is proportional to $P_{11}=0$ or $P_{12}=0$, and similarly for the $h_{1}$. The $a_{1}$ is stable and the only allowed $h_{1}$ decay for $m_{h_{1}} \approx 60 \mathrm{GeV}$ is $h_{1} \rightarrow a_{1} a_{1}$. The singlet sector is decoupled from the SM sector.

Furthermore, for the case $\lambda=0$, the singlet sector is decoupled from the MSSM sector. One neutralino is pure singlino whose mass, at tree level, is related to the $a_{1}$ mass by $m_{\chi}=-2 m_{a_{1}}^{2} / 3 A_{\kappa}[15,17]$. For $m_{a_{1}} \approx 10 \mathrm{GeV}$ and $\left|A_{\kappa}\right|$ of $\mathcal{O}(1) \mathrm{GeV}$, consistent with a slightly broken PQ symmetry, this yields $m_{\chi} \approx 60 \mathrm{GeV}$. In this study we identify the singlino as the LSP $\chi_{1}$. Denoting $N_{i j}^{2}$ as the $j$ th component of $\chi_{i}$, where $j=1,2,3,4,5$ corresponds to bino, wino, $u$ higgsino, $d$ higgsino, and singlino respectively. Neutralinos heavier than the singlino LSP have a zero singlino component, $N_{15}=1$ and all other $N_{i 5}$ vanish. No heavier neutralino can decay to the singlino since the coupling is proportional to $N_{i 5}=0$ for $i>1$. The NLSP $\chi_{2}$ is stable for conserved $\mathrm{R}$ parity.

However, when the singlet is allowed a weak coupling to the doublets $(\lambda \approx 0)$, small mixing between the singlet sector and the SM and MSSM sectors is possible. In this case the $a_{1}$ may couple to SM pairs so the $a_{1}$ is no longer stable. For $m_{a_{1}} \approx 10 \mathrm{GeV}, a_{1} \rightarrow \tau^{+} \tau^{-}$dominates, with decays to gluon and light quark pairs subdominant. For $m_{h_{1}} \approx 60 \mathrm{GeV}$, $h_{1} \rightarrow a_{1} a_{1}$ remains dominant with decays to SM pairs, notably $h_{1} \rightarrow b \bar{b}$, subdominant. Furthermore, the $\chi_{1}$ can be produced from heavier neutralino decays since singlino mixing with doublinos is allowed. Then the NLSP $\chi_{2}$ and NNLSP $\chi_{3}$ are no longer stable against decay to $\chi_{1}$. Above the threshold $m_{\chi_{2}}=m_{\chi_{1}}+m_{a_{1}} \approx 70 \mathrm{GeV}, \chi_{2} \rightarrow \chi_{1} a_{1}$ dominates while below it $\chi_{2} \rightarrow \chi_{1} Z^{\star}$ dominates. In the latter case, the decay may occur outside the LHC detector effective tracking volume for $\lambda$ of $\mathcal{O}\left(10^{-2}\right)$ or less [31]. Above the threshold $m_{\chi_{3}}=m_{\chi_{1}}+m_{h_{1}} \approx 120 \mathrm{GeV}, \chi_{3} \rightarrow \chi_{1} h_{1}$ dominates while below it $\chi_{3} \rightarrow \chi_{1} a_{1}$ and $\chi_{3} \rightarrow \chi_{1} Z^{\star}$ dominate.

Further information as regards $\lambda$ and $\kappa$ can be extracted from the $h_{1,2}$ sum rule [17]

$m_{h_{1}}^{2}+m_{h_{2}}^{2} \approx m_{Z}^{2}+\frac{1}{2} \kappa v_{S}\left(4 \kappa v_{s}+\sqrt{2} A_{\kappa}\right)$

where $v_{s} \equiv \sqrt{2} \mu_{e f f} / \lambda$. For $m_{h_{1}}=60 \mathrm{GeV}, m_{h_{2}}=125 \mathrm{GeV}$ and $\mu_{e f f}=300 \mathrm{GeV}$, the sum rule yields $|\kappa / \lambda| \approx 0.176$.

To summarize, we assume an effective MSSM with mostly singlino LSP $\chi_{1}$ and $m_{\chi_{1}} \approx 60 \mathrm{GeV}$. The $a_{1}$ and $h_{1}$ are mostly singlet with dominant decays to SM $\tau$ pairs and $a_{1}$ pairs, respectively. The $a_{1}, h_{1}$, and $\chi_{1}$ can be produced in neutralino decays. For $m_{\chi_{2}} \approx 70 \mathrm{GeV}$ or below and $\lambda<\mathcal{O}\left(10^{-2}\right)$, the $\chi_{2}$ decays outside of the effective tracking volume. For $m_{\chi_{3}} \approx 120 \mathrm{GeV}$ or above, $\chi_{3} \rightarrow \chi_{1} h_{1}$ is dominant. Finally, the $h_{1,2}$ mass sum rule yields $|\kappa / \lambda| \approx 0.176$ for $\mu_{\mathrm{eff}}=$ $300 \mathrm{GeV}$. These considerations, together with naturalness, inform the parameter ranges in the scan described in the next section.

\section{Parameter scan}

The parameter scan is performed with NMSSMTools4.4.0 [32-37], probing $10^{8}$ random points. We trade the soft trilinear parameters $A_{\lambda}, A_{\kappa}, A_{t}$ for $m_{P}, m_{A}, X_{t}$, defined by $[15,16]$

$m_{A}^{2}=\frac{\lambda v_{s}}{\sin 2 \beta}\left(\sqrt{2} A_{\lambda}+\kappa v_{s}\right)$ 
Table 1 NMSSM parameters and their scan ranges. Additionally, $\kappa$ is constrained to satisfy $0.125 \lambda<|\kappa|<0.225 \lambda$. The point $h_{60}(\kappa=$ 0.006088 and $A_{\kappa}=-1.087 \mathrm{GeV}$ ) is taken from points surviving the scan and is described in Sect. 5

\begin{tabular}{lll}
\hline Parameter & Range/Value & $h_{60}$ \\
\hline$\lambda$ & $(0,0.1]$ & 0.03505 \\
$\kappa$ & {$[-0.01,0.01]$} & 0.006088 \\
$m_{A}$ & {$[500,1500] \mathrm{GeV}$} & $1068 . \mathrm{GeV}$ \\
$m_{P}$ & {$[9.9,10.5] \mathrm{GeV}$} & $10.25 \mathrm{GeV}$ \\
$\mu_{e f f}$ & {$[100,300] \mathrm{GeV}$} & $166.7 \mathrm{GeV}$ \\
$\tan \beta$ & {$[1,30]$} & 15.49 \\
$M_{1}$ & $\frac{1}{2} M_{2}$ & $80.73 \mathrm{GeV}$ \\
$M_{2}$ & {$[100,300] \mathrm{GeV}$} & $161.5 \mathrm{GeV}$ \\
$M_{3}$ & $3 M_{2}$ & $484.4 \mathrm{GeV}$ \\
$X_{t}$ & {$\left[0.8 X_{t}^{\max }, 1.8 X_{t}^{\max }\right]$} & $1378 . \mathrm{GeV}$ \\
$m_{\tilde{Q} 3_{L}}$ & {$[350,550] \mathrm{GeV}$} & $546.9 \mathrm{GeV}$ \\
$m_{\tilde{U} 3_{R}}$ & $m_{Q 3}$ & $546.9 \mathrm{GeV}$ \\
\hline
\end{tabular}

$m_{P}^{2}=-\frac{3}{\sqrt{2}} \kappa v_{s} A_{\kappa}$

$X_{t}=A_{t}-\mu_{\text {eff }} / \tan \beta$

Here $m_{A}\left(m_{P}\right)$ is the diagonal component of the CP odd doublet (singlet) mass matrix and $X_{t}$ is the stop mixing parameter.

The parameters scanned are $\lambda, \kappa, m_{A}, m_{P}, \mu_{\mathrm{eff}}, \tan \beta, M_{2}$, $X_{t}$, and $m_{Q_{3}}$. We fix the gaugino masses $M_{1}$ and $M_{3}$ with the unification constraints $M_{1}=\frac{1}{2} M_{2}$ and $M_{3}=3 M_{2}$. We further assume $m_{Q_{3}}=m_{U_{3}}$. All other squark and soft trilinear parameters are fixed to $1500 \mathrm{GeV}$, and the slepton mass parameters are fixed to $200 \mathrm{GeV}$. See Table 1 for scanned parameter ranges.

Motivated by the PQ symmetric NMSSM, we scan small $\kappa$ and $A_{\kappa}$ or equivalently, from Eq. 4, small $\kappa$ and small $m_{P}$. The lower range bound of $m_{P}(9.9 \mathrm{GeV})$ is informed by the anomalous muon magnetic moment study [25], while the upper bound $(10.5 \mathrm{GeV})$ is informed by the $\eta_{b}$ mass spectrum study [26]. Then $\kappa$ is scanned in the range $-0.01<\kappa<0.01$ and is also required to satisfy $0.125 \lambda<|\kappa|<0.225 \lambda$ since this requires $m_{h_{1}} \approx 60 \mathrm{GeV}$ within several $\mathrm{GeV}$. We scan moderately small $\lambda$ in the range $0<\lambda<0$. 1 . Since the $h_{125}$ signal strength constraints are applied in the scan, $m_{A}$ is allowed to go into the effective MSSM decoupling limit $m_{A} \gg m_{Z}$ to accommodate the SM-like couplings of the $h_{125}$.

The neutralino and chargino masses are largely determined by $\mu_{\text {eff }}, M_{1}$ and $M_{2}$, which, from naturalness considerations are bounded above in the scan by $300 \mathrm{GeV}$ [12]. At tree level, the stop masses are $m_{\tilde{t}_{1}, \tilde{t}_{2}}^{2}=m_{Q_{3}}^{2}+m_{t}^{2} \pm m_{t} X_{t}$ for $m_{Q_{3}}=m_{U_{3}}$ [15]. Naturalness informs the $m_{Q_{3}}$ range since light stops are compatible with small fine tuning.
The tree level Higgs mass in the MSSM is bounded by $m_{h}^{2}<m_{Z}^{2} \cos ^{2} 2 \beta$, requiring a large loop correction for the $h_{125}$. In the NMSSM the upper bound on $m_{h}^{2}$ has an additional $\mathcal{O}\left(\lambda^{2} v^{2}\right)$ term. The stop mixing parameter $X_{t}$ partly determines the one loop correction [12]:

$\delta m_{h}^{2}=\frac{3 G_{F}}{\sqrt{2} \pi^{2}} m_{t}^{4}\left[\log \left(\frac{m_{\tilde{t}}^{2}}{m_{t}^{2}}\right)+\frac{X_{t}^{2}}{m_{\tilde{t}}^{2}}\left(1-\frac{X_{t}^{2}}{12 m_{\tilde{t}}^{2}}\right)\right]$

where the parameter $m_{\tilde{t}}$ is defined by $m_{\tilde{t}}^{2} \equiv \frac{1}{2}\left(m_{\tilde{t}_{1}}^{2}+m_{\tilde{t}_{2}}^{2}\right)$. The correction is strongly dependent on the top mass $m_{t}$. In the scan $m_{t}=172.5 \mathrm{GeV}$.

To allow the large correction required by the $h_{125}$, but with small $m_{\tilde{t}}$ required by Natural SUSY, the stop mixing $X_{t}$ is allowed to contribute up to its maximal possible correction at $X_{t}^{\max }=\sqrt{6} m_{\tilde{t}}$. In the scan NMSSMTools4 calculates the Higgs mass spectrum at one-loop level including external momentum for self-energies and two-loop level excluding external momentum [38,39].

\section{Surviving points}

The suite of constraints imposed by NMSSMTools4 while scanning includes experimental results from a wide variety of sources, including:

- Anomalous muon magnetic moment $\Delta a_{\mu}$ measured by BNL E821 [40]

- DM relic density $\Omega_{D M} \hbar^{2}$ measured by Planck [41], direct DM exclusion by LUX [42]

- $B$ Physics. $b \rightarrow s \gamma, B \rightarrow X_{s} \mu^{+} \mu^{-}, B^{+} \rightarrow \tau^{+} \nu, B_{s} \rightarrow$ $\mu^{+} \mu^{-}, \Upsilon(1 s) \rightarrow a \gamma, \eta_{b}(1 s)$

- Higgs. LHC $h_{125}$, LEP $e^{+} e^{-} \rightarrow Z h$, Tevatron/LHC $t \rightarrow$ $b H^{+}$, NMSSM searches

- SUSY. Tevatron/LHC $\chi^{+}, \tilde{q}, \tilde{g}, \tilde{e}, \tilde{\mu}, \tilde{\tau}$ mass constraints, $\tilde{t} \rightarrow b \ell \tilde{v}, \chi^{0} c$, and $\tilde{b} \rightarrow \chi^{0} b$

Loose constraints imposed during the scan require $m_{h_{2}} \approx$ 125 within $3 \mathrm{GeV}$ and impose an upper bound on each $h_{125}$ signal strength $\chi^{2}$, calculated as in [43]. Of the $10^{8}$ points scanned, 42 survive the constraints imposed during the scan.

Constraints are tightened after the scan. The low mass Higgs sector must satisfy

$$
\begin{aligned}
9.9 & <m_{a_{1}}<10.5 \mathrm{GeV} \\
50 & <m_{h_{1}}<70 \mathrm{GeV} \\
122 & <m_{h_{2}}<128 \mathrm{GeV}
\end{aligned}
$$

Finally, the sum of $h_{125}$ signal strength $\chi^{2}$ are required to satisfy $\sum_{i} \chi_{i}^{2}<13$. Of the 42 points surviving the scan constraints, 15 points survive these final constraints. 
In order to demonstrate the naturalness of the surviving points, we examine the fine tuning metric $F_{\max } \equiv$ $\max _{a \in A}\left(\frac{\partial\left(\log m_{Z}^{2}\right.}{\partial\left(\log a^{2}\right)}\right)$ calculated by NMSSMTools4. This metric yields the largest fine tuning over fundamental parameter set $A$. Surviving points have small fine tuning, $5<F_{\max }<$ 10 , light stops $300<m_{\tilde{t}_{1}}<400 \mathrm{GeV}$ and light gluinos $500<m_{\tilde{g}}<650 \mathrm{GeV}$. While agreement is not universal on which $F_{\max }$ values characterize low fine tuning [19], studies have considered $F_{\max }$ of order $\mathcal{O}\left(10^{2}\right)$ to be typical for the NMSSM [44] and $\mathcal{O}\left(10^{1}\right)$ to be low fine tuning [22-24,45]. A recent study seeking to establish naturalness as objective, model-independent and predictive concludes that a SUSY model with $F_{\max }<30$ is natural, while one with $F_{\max }<10$ is stringently natural [46].

\section{Benchmark $\mathbf{h}_{60}$}

It has been noted that points surviving the scan represent a Natural NMSSM with slightly broken PQ symmetry. They also exhibit a light pseudoscalar Higgs with $m_{a_{1}} \approx 10 \mathrm{GeV}$, a light scalar Higgs with $m_{h_{1}} \approx 60 \mathrm{GeV}$, a singlino LSP DM candidate with $m_{\chi_{1}} \approx 60 \mathrm{GeV}$ annihilating via $\chi_{1} \chi_{1} \rightarrow b \bar{b}$, and a light stop with $m_{\tilde{t}_{1}} \approx 350 \mathrm{GeV}$.

The benchmark point $h_{60}$ satisfies the threshold criterion $m_{\chi_{3}}>m_{h_{1}}+m_{\chi_{1}}$ with the largest branching ratio for $\chi_{3} \rightarrow \chi_{1} h_{1}$ of all surviving points in the scan. The lowest branching ratio for this decay in the surviving points which reach threshold is $65 \%$, while the highest is $80 \%$. This ensures production of $a_{1}$ from $h_{1} \rightarrow a_{1} a_{1}$ in stop pair events with $\tilde{t}_{1} \rightarrow \chi_{2}^{+} b \rightarrow \chi_{3} W b$ or $\tilde{t}_{1} \rightarrow \chi_{3} t$. See the last column of Table 1 for the numerical values of the parameters which define $h_{60}$. See Fig. 1, generated with PySLHA [47] using the SUSY Les Houches Accord (SLHA) [48,49] file produced by NMSSMTools4, for the mass spectrum and decays in $h_{60}$.

For components $P_{1 j}, S_{1 j}, N_{i j}$ of the $a_{1}, h_{1}, \chi_{1}, \chi_{2}$ and $\chi_{3}$ in $h_{60}$ see Table 2 . The LSP $\chi_{1}$ is dominantly singlino, while the $a_{1}$ and $h_{1}$ are dominantly singlet. The $\chi_{1}$ mixing with doublinos and gauginos is small, as is the mixing of the $a_{1}$ and $h_{1}$ with the doublets. The phenomenology of a singlino LSP at the LHC has been considered in [31,50-57]. The phenomenology of light stops in the NMSSM, and how they avoid exclusion at the LHC, has been recently considered in [58].

For the numerical values of the masses and dominant branching ratios of the low mass spectrum of the $h_{60}$ benchmark, see Table 3. The $a_{1}$ and $h_{1}$ of the benchmark avoids the LHC search exclusion for straightforward reasons. Both ATLAS and CMS have searched for gluon fusion $g g \rightarrow a \rightarrow$ $\mu^{+} \mu^{-}$but critically omit the $\Upsilon$ region and therefore cannot exclude $m_{a_{1}} \approx 10 \mathrm{GeV}[59,60]$. ATLAS has searched for

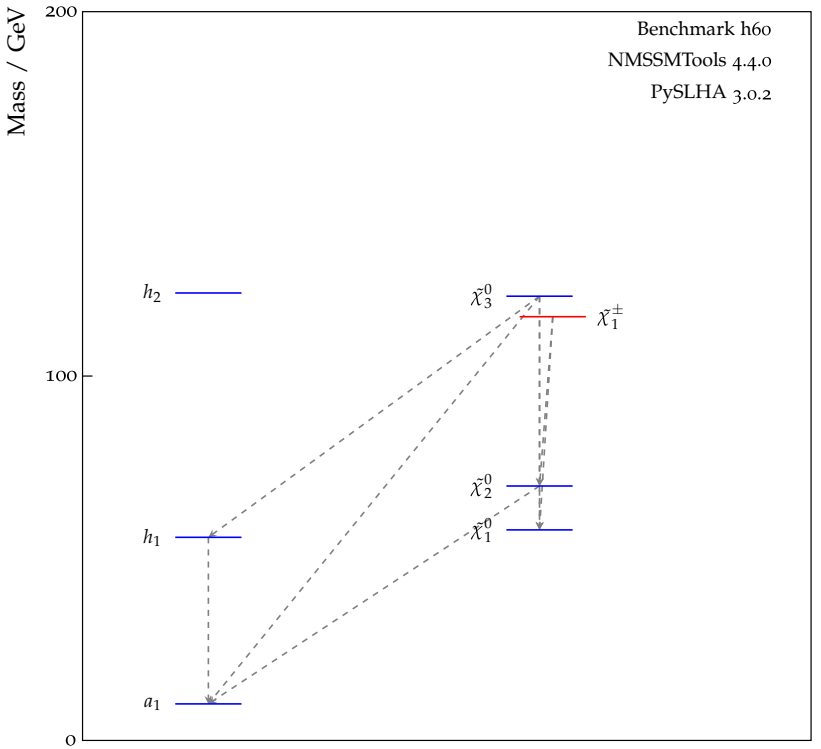

Fig. 1 Masses and decays for the lighter $h_{60}$ benchmark spectrum. Only decays with branching ratios larger than $5 \%$ are shown. Note that the $h_{2}$ is mostly decoupled

Table 2 Doublet and singlet components of the $a_{1}, h_{1}$, and gaugino and singlino components of the $\chi_{1}, \chi_{2}, \chi_{3}$ in $h_{60}$

\begin{tabular}{lrrrrl}
\hline Component & \multicolumn{1}{c}{$a_{1}$} & \multicolumn{1}{c}{$h_{1}$} & \multicolumn{1}{c}{$\chi_{1}$} & \multicolumn{1}{c}{$\chi_{2}$} & \multicolumn{1}{l}{$\chi_{3}$} \\
\hline$P_{11} / S_{11} / N_{i 1}$ & -0.002 & -0.006 & 0.151 & 0.882 & 0.401 \\
$P_{12} / S_{12} / N_{i 2}$ & 0.000 & -0.130 & -0.054 & -0.153 & 0.679 \\
$P_{13} / S_{13} / N_{i 5}$ & 1.000 & 0.992 & 0.981 & -0.189 & 0.045 \\
\hline
\end{tabular}

Table 3 Masses and the two dominant decays of the lighter part of the $h_{60}$ benchmark point spectrum obtained by NMSSMTools4. The column titled "Range" is the mass range of the points surviving all scan constraints

\begin{tabular}{llcll}
\hline & Range & Mass $(\mathrm{GeV})$ & $\mathrm{BR} 1(\%)$ & $\mathrm{BR} 2(\%)$ \\
\hline$a_{1}$ & {$[9.9,10.4]$} & 10.0 & $\tau^{+} \tau^{-}(81)$ & $g g(16)$ \\
$h_{1}$ & {$[53,59]$} & 55.7 & $a_{1} a_{1}(72)$ & $b \bar{b}(23)$ \\
$h_{2}$ & {$[122,123]$} & 122.8 & $b \bar{b}(65)$ & $W W^{\star}(17)$ \\
$\chi_{1}$ & {$[54,60]$} & 57.8 & - & - \\
$\chi_{2}$ & {$[57,76]$} & 69.8 & $\chi_{1} a_{1}(75)$ & $\chi_{1} Z^{\star}(25)$ \\
$\chi_{3}$ & {$[107,136]$} & 121.9 & $\chi_{1} h_{1}(80)$ & $\chi_{2} Z^{\star}(10)$ \\
$\chi_{4}$ & {$[166,209]$} & 179.5 & $\chi_{2} Z(80)$ & $\chi_{1} Z(14)$ \\
$\chi_{5}$ & {$[225,254]$} & 236.6 & $\chi_{1}^{+} W(60)$ & $\tilde{v} v, \tilde{\ell} \ell(38)$ \\
$\chi_{1}^{+}$ & {$[104,132]$} & 116.3 & $\chi_{2} W^{\star}(78)$ & $\chi_{1} W^{\star}(22)$ \\
$\chi_{2}^{+}$ & {$[225,255]$} & 237.1 & $\ell \tilde{v}, \tilde{\ell} v(40)$ & $\chi_{3} W(38)$ \\
$\tilde{t}_{1}$ & {$[313,391]$} & 335.6 & $\chi_{2}^{+} b(75)$ & $\chi_{3} t(15)$ \\
\hline
\end{tabular}

gluon fusion $g g \rightarrow h \rightarrow a a$ for $2 m_{\tau}<m_{a}<2 m_{B}$ but does not report limits for $m_{h}<100 \mathrm{GeV}$ [61]. CMS has searched for the same channel but only reports limits for $m_{h}>90 \mathrm{GeV}$ with $m_{a}<2 m_{\tau}$ [62] or for the $h_{125}$ with $4<m_{a}<8 \mathrm{GeV}$ 
[63]. More decisively, the gluon fusion cross sections for $a_{1}$ and $h_{1}$ production in the benchmark are greatly reduced relative to the $h_{125}$.

In the neutralino and chargino sector, both ATLAS and CMS have studied $\chi_{2} \chi_{1}^{+}$production [64-66]. For example, the searches which assume decays to sleptons or to bosons also assume that $m_{\chi_{1}^{+}}=m_{\chi_{2}}$, motivated by models with a bino-like $\chi_{1}$ and wino-like $\chi_{2}$ and $\chi_{1}^{+}$. But in $h_{60}$ the $\chi_{1}$ is singlino, and manifestly $m_{\chi_{1}^{+}} \neq m_{\chi_{2}}$. The $\chi_{2} \chi_{1}^{+}$searches which assume dominant decays to sleptons cannot exclude $h_{60}$ where $m_{\tilde{\ell}}>m_{\chi_{1}^{+}}, m_{\chi_{2}}$. Such searches might be sensitive to $\chi_{5} \chi_{2}^{+}$events, but here the cross section is reduced and the final states are more complex. Of the searches which assume dominant decays to bosons, only the $W \chi_{1} Z \chi_{1}$ final state case applies. In this case both $W$ and $Z$ are very far off mass shell in $h_{60}$, in which case it is unlikely that the exclusion can apply.

In the stop sector, both ATLAS and CMS report exclusion. For a summary of the ATLAS results, see $[67,68]$. For a bibliography of CMS results see [69]. No exclusion is given for the NMSSM, however, and exclusion for simplified models cannot be easily interpreted in the NMSSM context. For example, the analyses which assume $\tilde{t} \rightarrow t \chi_{1}$ with $100 \%$ branching ratio cannot exclude $h_{60}$, for which this branching ratio is $\mathcal{O}\left(10^{-3}\right)$. $h_{60}$ does contain $\tilde{t} \rightarrow t \chi_{3}$ with a branching ratio $\mathcal{O}\left(10^{-1}\right)$, but the subsequent $\chi_{3}$ decay produces a much more complex final state with less missing energy than assumed by the searches. The stop pair searches which assume $\tilde{t} \rightarrow b \chi_{1}^{+}$with $100 \%$ branching ratio assume very specific cases of mass relationships between stops, neutralino and charginos which do not hold in $h_{60}$. Moreover they assume $\chi_{1}^{+} \rightarrow W \chi_{1}$. But the dominant branching in $h_{60}$ is $\chi_{1}^{+} \rightarrow W^{\star} \chi_{2} \rightarrow W^{\star} Z^{\star} \chi_{1}$, producing less missing energy, and two gauge bosons which are very far off mass shell in comparison to the search assumptions.

In order to evaluate quantitatively the $h_{60}$ exclusion at LHC Run 1, we run all 16 (3) presently validated ATLAS (CMS) analyses in Checkmate1.2.2 [70-75] on generated $h_{60}$ events. Event simulation of the gluino, stop and chargino/neutralino pair production is carried out with Pythia8.205 [76,77]. The SLHA file produced by NMSSMTools4 for $h_{60}$ is used with Pythia8, which features a dedicated NMSSM model with functionality for SLHA input.

See Table 4 for the exclusion $r_{\max }$, the ratio of the $95 \%$ confidence level lower limit on the $h_{60}$ signal presence to the measured $95 \%$ confidence level limit, of the analyses with maximum sensitivity to $h_{60}$ chargino/neutralino, stop and gluino pair production. Only for gluino pair production is $r_{\max }>1$, indicating that both ATLAS and CMS have ruled out a gluino with $m_{\tilde{g}} \approx 611 \mathrm{GeV}$ in $h_{60}$ but neither has ruled out the stop and chargino/neutralino sectors of $h_{60}$. However, since $m_{\tilde{g}}$ is determined by the gaugino mass $M_{3}$, which can be easily increased without otherwise impacting
Table 4 Maximum exclusion $r_{\max }$ determined by Checkmate 1 of the analyses most sensitive to $h_{60}$ of all the validated ATLAS and CMS Run 1 analyses. Note that neither stop nor chargino/neutralino pair production is ruled out for $h_{60}$

\begin{tabular}{lrll}
\hline Sample & \multicolumn{1}{c}{$r_{\max }$} & Analysis & $\int d t \mathcal{L}\left(\mathrm{fb}^{-1}\right)$ \\
\hline$\chi \bar{\chi}$ & 0.6 & atlas_conf_2013_035 & 20.7 \\
$\tilde{t_{1}} \tilde{\bar{t}}$ & 0.5 & atlas_conf_2013_061 & 20.1 \\
$\tilde{g} \tilde{g}$ & 12.5 & atlas_conf_2013_061 & 20.1 \\
$\chi \bar{\chi}$ & 0.1 & cms_1303_2985 & 11.7 \\
$\tilde{t_{1}} \tilde{\tilde{t_{1}}}$ & 0.6 & cms_1502_06031 & 19.5 \\
$\tilde{g} \tilde{g}$ & 1.2 & cms_1303_2985 & 11.7 \\
\hline
\end{tabular}

the lower energy $h_{60}$ phenomenology, we simply assume $m_{\tilde{g}} \approx 855 \mathrm{GeV}$ or greater since this reduces the gluino pair production cross section by a factor of 13 relative to $h_{60}$.

Note that $r_{\max }=0.6$ for cms_1502_06031, which exhibits a $3 \sigma$ excess in the low dilepton mass region [78]. If the $h_{60}$ stop mass is reduced such that the stop pair production cross section is enhanced by a factor of 1.5 , then this analysis becomes sensitive to $h_{60}$ with the reduced $m_{\tilde{t}} \approx 315 \mathrm{GeV}$.

\section{Collider signature}

Since the stop is relatively light in $h_{60}$, the cross section for pair production is large and makes cascade production of the $a_{1}$ and $h_{1}$ accessible at the LHC. Gluon fusion production of $a_{1}$ and $h_{1}$ is less promising. The reduced $t t h_{1}\left(t t a_{1}\right)$ coupling, which appears in the gluon fusion top loop, is of order $\mathcal{O}\left(10^{-1}\right)\left(\mathcal{O}\left(10^{-5}\right)\right)$ relative to the $\mathrm{SM} t t H_{S M}$ coupling for a SM Higgs boson of the same mass.

From Table 3 the decays $\tilde{t}_{1} \rightarrow \chi_{2}^{+} b, \chi_{2}^{+} \rightarrow \chi_{3} W, \chi_{3} \rightarrow$ $\chi_{1} h_{1}$, and $h_{1} \rightarrow 2 a_{1}$ proceed with branching ratios of 75 , 38,80 , and $72 \%$ respectively, while $\tilde{t}_{1} \rightarrow \chi_{3} t$ proceeds with branching ratio of $15 \%$. This makes stop pair production with $\tilde{t}_{1} \rightarrow \chi_{2}^{+} b \rightarrow \chi_{3} W b$ or $\tilde{t}_{1} \rightarrow \chi_{3} t$ and $\chi_{3} \rightarrow \chi_{1} h_{1} \rightarrow \chi_{1} 2 a_{1}$ promising channels for discovery if the $a_{1}$ can be successfully reconstructed, for example in the relatively rare but very clean $a_{1} \rightarrow \mu^{+} \mu^{-}$channel. In $h_{60}$ this decay proceeds with a branching ratio of $0.3 \%$. These remarks also apply to stop pairs produced in gluino pair production with $\tilde{g} \rightarrow \tilde{t}_{1} t$.

In $h_{60}$ stop pair production, the cascade dominantly contains two top quarks. In gluino pair production it dominantly contains four top quarks. These are strong handles on any potential background. Some top pair $t \bar{t}$ background may be irreducible, but other backgrounds should be negligible.

We now describe a targeted study of the sensitivity to $h_{60}$ at the LHC. Signal events are generated with Pythia8 as described in Sect. 5. Background $t \bar{t}$ events are also generated in Pythia8. Fast detector simulation is performed with Delphe3.2.0 [71]. The Delphes3 detector card for CMS is 
modified to reproduce the tight electron, tight muon and $b$ tag efficiencies reported by CMS [79-81]. The signal selection seeks the decay $a_{1} \rightarrow \mu^{+} \mu^{-}$in gluino and stop pair events and employs a standard selection for semileptonic top pair events, together with a selection for $a_{1} \rightarrow \mu^{+} \mu^{-}$, in which one top quark decays via $t \rightarrow b W \rightarrow b \ell v$ and the other via $t \rightarrow b W \rightarrow b q q^{\prime}$. The requirements for the Run 1 analysis are these:

- Exactly one tight electron with $E_{T}>25 \mathrm{GeV}$ and no isolation requirement

- Missing transverse energy $E_{T}^{\text {miss }}>85 \mathrm{GeV}$

- Four or more jets with $E_{T}>20 \mathrm{GeV}$, at least two of which are $b$-tagged

- Two or more tight muons with $p_{T}>2 \mathrm{GeV}$, no isolation requirement and $d_{0} / \sigma_{d_{0}}<5$

- Zero net charge and $9.7<m_{\mu^{+} \mu^{-}}<10.3 \mathrm{GeV}$ in the leading and subleading muons

The $a_{1}$ candidate is then reconstructed from the leading and subleading muons. The muon azimuthal impact parameter

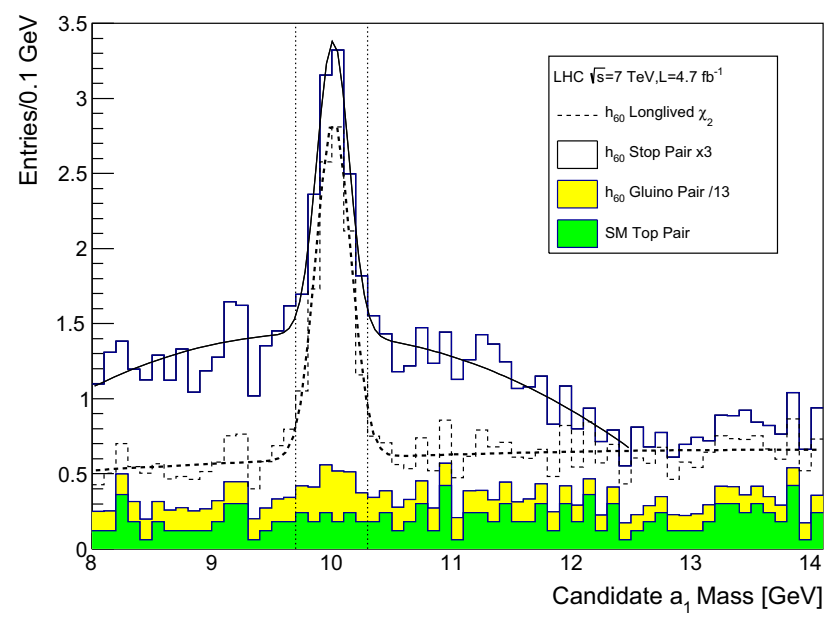

Fig. 2 Reconstructed candidate $a_{1} \rightarrow \mu^{+} \mu^{-}$mass distribution after full signal selection assuming $h_{60}\left(\times 3\right.$ for $\tilde{t_{1}} \tilde{\overline{t_{1}}}$ and $/ 13$ for $\left.\tilde{g} \tilde{g}\right)$ at the LHC for $\sqrt{s}=7 \mathrm{TeV}$ and $\int d t \mathcal{L}=4.7 \mathrm{fb}^{-1}$. Also shown (dashed) is a variation of $h_{60}$ in which the $\chi_{2}$ decays outside of the effective tracking volume. The fits employ a Gaussian signal model and a polynomial background model

Table 5 NLO cross sections for $\tilde{t}_{1} \tilde{\bar{t}}_{1}$ and $t \bar{t}$ production at the LHC in Runs $1(\sqrt{s}=7,8 \mathrm{TeV})$ and $2(\sqrt{s}=14 \mathrm{TeV})$. Also shown are the expected yields for peaking events $\left(N_{p}\right)$, yields for nonpeaking events $\left(N_{n}\right)$ and signal significances after the full signal selection described in significance requirement $d_{0} / \sigma_{d_{0}}<5$ ensures that the muons are consistent with prompt production.

For the Run 2 analysis, we assume $\sqrt{s}=14 \mathrm{TeV}$ and $\int d t \mathcal{L}=300 \mathrm{fb}^{-1}$. We use the Delphes 3 simulation with mean pileup 50. The selection is identical to the Run 1 analysis except that the electron, jet, and muon thresholds are raised to 30,30 and $4 \mathrm{GeV}$, respectively.

After full signal selection, the SM top background is nearly negligible. Multiple jet events produced by QCD have not been simulated, but with the nominal selection this background is expected to be very small. In data, the nonpeaking $h_{60}$ events in the candidate $a_{1}$ distribution can be mistaken for QCD multijets events, however, so these are considered background in the significance calculation. In the SM top background, the candidate $a_{1}$ muons originate from $\tau$ lepton, $D$ meson or $B$ meson decays. In the nonpeaking $h_{60}$ events, they originate either from SM $\tau, B$ or $D$ decay or from NMSSM $a_{1} \rightarrow \tau_{\mu} \tau, \chi_{2} \rightarrow \chi_{1} \mu^{+} \mu^{-}, \chi^{+} \rightarrow \chi \mu \nu$, or $\chi^{+} \rightarrow \mu \tilde{\nu}$.

The proportion of peaking to nonpeaking signal events is sensitive to the details of $h_{60}$. For example, if the slepton masses are raised above the threshold for decay from $\chi_{2}^{+}$, then the peaking signal is enhanced and the nonpeaking signal is reduced. Similarly, if the branching ratio for $\chi_{2} \rightarrow \chi_{1} a_{1}$ is raised at the expense of $\chi_{2} \rightarrow \chi_{1} Z^{\star}$, the peaking signal is enhanced and the nonpeaking signal is reduced. Finally, if the $\chi_{2}$ width is sufficiently small, its decay vertices may lie outside the effective tracking volume, making nonpeaking background from $\chi_{2}$ effectively invisible.

Pythia8 is a leading order generator, but next to leading order cross sections obtained by the LHC SUSY Working Group $[82,83]$ are used to normalize the event yields. See Figure 2 for the reconstructed $a_{1}$ mass distribution after full signal selection, where the distribution for a variation of $h_{60}$ in which the $\chi_{2}$ decays outside of the tracking volume is also shown. See Table 5 for expected peaking and nonpeaking event yields and signal significances after full selection for Runs 1 and 2 at the LHC. A targeted $h_{60}$ signal selection yields sensitivity even at the LHC Run 1.

the text. For $\sqrt{s}=7 \mathrm{TeV}$, we show in parentheses the yields and the significance for a variation of $h_{60}$ in which the $\chi_{2}$ decays outside of the effective tracking volume

\begin{tabular}{|c|c|c|c|c|c|c|c|c|c|}
\hline \multirow[t]{2}{*}{ Process } & \multicolumn{3}{|c|}{ Run 1: $\mathcal{L}=5 \mathrm{fb}^{-1}$} & \multicolumn{3}{|c|}{ Run 1: $\mathcal{L}=20 \mathrm{fb}^{-1}$} & \multicolumn{3}{|c|}{ Run 2: $\mathcal{L}=300 \mathrm{fb}^{-1}$} \\
\hline & $\sigma_{7 T}(\mathrm{pb})$ & $N_{n}$ & $N_{p}$ & $\sigma_{8 T}(\mathrm{pb})$ & $N_{n}$ & $N_{p}$ & $\sigma_{14 T}(\mathrm{pb})$ & $N_{n}$ & $N_{p}$ \\
\hline $\mathrm{SM} t \bar{t}$ & 173.6 & 1.6 & 0 & 247.7 & 8.5 & 0 & 966.0 & 286.0 & 0 \\
\hline$h_{60} \tilde{t}_{1} \tilde{\bar{t}}_{1}$ & 0.7 & $2.6(0.8)$ & $2.2(2.5)$ & 1.1 & 20.1 & 19.2 & 5.9 & 892.5 & 647.9 \\
\hline$S / \sqrt{B}$ & $1.0(1.6)$ & & & 3.6 & & & 18.9 & & \\
\hline
\end{tabular}


The advantages of the International Linear Collider (ILC) for studying low mass NMSSM Higgs bosons has been noted in [15]. At the ILC, the standard Higgsstrahlung production channel $e^{+} e^{-} \rightarrow Z h_{1}$ is suppressed in the NMSSM due to the measured SM-like $h_{125} \rightarrow Z Z^{\star}$ signal strength and the NMSSM coupling sum rule $\sum_{1=1}^{3} \xi_{Z Z h_{i}}^{2}=1$ [14]. Instead, in $h_{60}$ we note the possibility of resonant production $e^{+} e^{-} \rightarrow a_{1} h_{1}$, with cross section of several hundred picobarns at $\sqrt{s}=m_{Z}$. For $\sqrt{s}=500 \mathrm{GeV}$, pair production of all neutralinos and all charginos is accessible with cross sections nearing a picobarn, as well as $a_{1} h_{1}$ and $Z h_{2}$ production cross sections of about a hundred femtobarns.

In $h_{60}$ the $Z a_{1} h_{1}$ coupling is small enough to have evaded LEP searches [84,85] but large enough to be produced copiously at the ILC running on the $Z$ pole. The ILC sensitivity to $h_{60}$ in operating scenarios described in [86] defined by beam polarization, luminosity, and $\sqrt{s}$ and will be evaluated in a forthcoming companion study.

\section{Conclusion}

We have reviewed the motivation for a natural NMSSM with a slightly broken PQ symmetry and a lightly coupled singlet featuring a light singlet pseudoscalar $a_{1}$, a light singlet scalar $h_{1}$, and a light singlino LSP $\chi_{1}$ DM candidate annihilating via $\chi_{1} \chi_{1} \rightarrow b \bar{b}$.

A random parameter space scan is performed subject to a full suite of experimental constraints, including the anomalous muon magnetic moment, the DM relic density and collider searches. Surviving points are characterized by low fine tuning, and abundant pseudoscalar $a_{1}$ production identifies the benchmark point $h_{60}$. In addition this benchmark features light stops and light higgsinos, all characteristic of Natural SUSY.

The benchmark avoids the current LHC exclusion limits. For the $a_{1}$ and $h_{1}$, this is due to the reduced gluon fusion cross sections. For other SUSY searches, this is primarily due to the search assumption that stops, neutralinos, and charginos will decay directly to the LSP $\chi_{1}$ with no intermediate SUSY particles in the decay chain. But in the $h_{60}$ benchmark the $\chi_{1}$ is singlino and couples weakly to the rest of SUSY. Thus due to the light mass spectrum the decay chains can contain many intermediate SUSY particles, making the final states more complex with less missing energy than in the simplified search scenarios.

Finally, we report that the potentially fruitful discovery channels at the LHC for the benchmark considered are stop and gluino pair production with either $\tilde{t}_{1} \rightarrow \chi_{2}^{+} b \rightarrow \chi_{3} W b$ or $\tilde{t}_{1} \rightarrow \chi_{3} t$ and $\chi_{3} \rightarrow \chi_{1} h_{1} \rightarrow \chi_{1} a_{1} a_{1}$. We conclude with a fast simulation study that with a targeted signal selection the LHC may already be sensitive to $h_{60}$ in Run 1 . We have also pointed out the possibility to observe at the ILC reso- nant $e^{+} e^{-} \rightarrow a_{1} h_{1}$ at $\sqrt{s}=m_{Z}$ and pair production of all neutralinos and charginos at $\sqrt{s}=500 \mathrm{GeV}$.

Acknowledgments The author thanks M. Muhlleitner for discussion of the Higgs mass calculation in NMSSMTools4, Tao Liu for feedback on fine tuning and the PQ symmetric NMSSM, and the Alder Institute for High Energy Physics for financial support.

Open Access This article is distributed under the terms of the Creative Commons Attribution 4.0 International License (http://creativecomm ons.org/licenses/by/4.0/), which permits unrestricted use, distribution, and reproduction in any medium, provided you give appropriate credit to the original author(s) and the source, provide a link to the Creative Commons license, and indicate if changes were made.

Funded by $\mathrm{SCOAP}^{3}$.

\section{References}

1. G. Aad et al., Observation of a new particle in the search for the Standard Model Higgs boson with the ATLAS detector at the LHC. Phys.Lett. B716, 1-29 (2012). arXiv: 1207.7214

2. S. Chatrchyan et al., Observation of a new boson at a mass of 125 $\mathrm{GeV}$ with the CMS experiment at the LHC. Phys. Lett. B716, 30-61 (2012). arXiv:1207.7235

3. M.S. Carena, H.E. Haber, Higgs boson theory and phenomenology. Prog. Part. Nucl. Phys. 50, 63-152 (2003). arXiv:hep-ph/0208209

4. G. Aad et al., Combined measurement of the Higgs Boson Mass in $p p$ and $8 \mathrm{TeV}$ with the ATLAS and CMS Experiments. Phys. Rev. Lett. 114, 191803 (2015). arXiv: 1503.07589

5. V. Khachatryan et al., Precise determination of the mass of the Higgs boson and tests of compatibility of its couplings with the standard model predictions using proton collisions at 7 and $8 \mathrm{TeV}$. Eur. Phys. J. C 75(5), 212 (2015). arXiv: 1412.8662

6. G. Aad et al., Measurements of the Higgs boson production and decay rates and coupling strengths using $p p \mathrm{TeV}$ in the ATLAS experiment (2015). arXiv: 1507.04548

7. G. Aad et al., Study of the spin and parity of the Higgs boson in diboson decays with the ATLAS detector. 1506, 05669 (2015)

8. V. Khachatryan et al., Constraints on the spin-parity and anomalous HVV couplings of the Higgs boson in proton collisions at 7 and 8 TeV. Phys. Rev. D 92(1), 012004 (2015). arXiv:1411.3441

9. V. Khachatryan et al., Limits on the Higgs boson lifetime and width from its decay to four charged leptons (2015). arXiv:1507.06656

10. S.P. Martin, A supersymmetry primer (1997). hep-ph/9709356

11. M. Dine, Naturalness under. Stress 1501, 01035 (2015)

12. M. Papucci, J.T. Ruderman, A. Weiler, Natural SUSY Endures. JHEP, 1209, 035 (2012). arXiv: 1110.6926

13. M. Maniatis, The next-to-minimal supersymmetric extension of the standard model reviewed. Int. J. Mod. Phys. A25, 3505-3602 (2010). arXiv:0906.0777

14. U. Ellwanger, C. Hugonie, A.M. Teixeira, The next-to-minimal supersymmetric standard model. Phys. Rept. 496, 1-77 (2010). arXiv:0910.1785

15. D.J. Miller, R. Nevzorov, P.M. Zerwas, The higgs sector of the next-to-minimal supersymmetric standard model. Nucl. Phys. B 681(1-2), 3-30 (2004)

16. U. Ellwanger, Higgs Bosons in the next-to-minimal supersymmetric standard model at the LHC. Eur. Phys. J. C71, 1782 (2011). arXiv: 1108.0157

17. D.J. Miller, R. Nevzorov, The Peccei-Quinn axion in the next-to-minimal supersymmetric standard model (2003). arXiv:hep-ph/0309143 
18. L.J. Hall, T. Watari, Electroweak supersymmetry with an approximate U(1)(PQ). Phys. Rev. D 70, 115001 (2004). arXiv:hep-ph/0405109

19. C. Philip, Schuster and Natalia Toro. Persistent fine-tuning in supersymmetry and the NMSSM (2005). arXiv:hep-ph/0512189

20. R. Barbieri, L.J. Hall, A.Y. Papaioannou, D. Pappadopulo, V.S. Rychkov, An alternative NMSSM phenomenology with manifest perturbative unification. JHEP, 03, 005 (2008). arXiv:0712.2903

21. K. Choi, S.H. Im, K.S. Jeong, M.S. Seo, Higgs phenomenology in the Peccei-Quinn invariant NMSSM. JHEP, 1401, 072 (2014). arXiv: 1308.4447

22. R. Dermisek, J.F. Gunion, Escaping the large fine tuning and litthe hierarchy problems in the next to minimal supersymmetric model and $h \rightarrow a a$ decays. Phys. Rev. Lett. 95, 041801 (2005). arXiv:hep-ph/0502105

23. R. Dermisek, J.F. Gunion, The NMSSM Close to the R-symmetry limit and naturalness in $h \rightarrow a a$ decays for $m_{a}<2 m_{b}$. Phys. Rev. D 75, 075019 (2007). arXiv:hep-ph/0611142

24. R. Dermisek, J.F. Gunion, The NMSSM solution to the finetuning problem, precision electroweak constraints and the largest LEP Higgs event excess. Phys. Rev. D76, 095006 (2007). arXiv:0705.4387

25. J.F. Gunion, A Light CP-odd Higgs boson and the muon anomalous magnetic moment. JHEP, 0908, 032 (2009). arXiv:0808.2509

26. F. Domingo, U. Ellwanger, M.A. Sanchis-Lozano, Bottomoniom spectroscopy with mixing of eta(b) states and a light CP-odd Higgs. Phys. Rev. Lett. 103, 111802 (2009). arXiv:0907.0348

27. R. Dermisek, J.F. Gunion, New constraints on a light CP-odd Higgs boson and related NMSSM Ideal Higgs Scenarios. Phys. Rev. D81, 075003 (2010). arXiv: 1002.1971

28. J. March-Russell, J. Unwin, S.M. West, Closing in on asymmetric dark matter I: model independent limits for interactions with quarks. JHEP, 1208, 029 (2012). arXiv: 1203.4854

29. S. Schael et al., Search for neutral MSSM Higgs bosons at LEP. Eur. Phys. J. C47, 547-587 (2006). arXiv:hep-ex/0602042

30. F. Franke, H. Fraas, Neutralinos and Higgs bosons in the next-tominimal supersymmetric standard model. Int. J. Mod. Phys. A 12, 479-534 (1997). arXiv:hep-ph/9512366

31. U. Ellwanger, C. Hugonie, Neutralino cascades in the (M+1)SSM. Eur. Phys. J. C 5, 723-737 (1998). arXiv:hep-ph/9712300

32. U. Ellwanger, J.F. Gunion, C. Hugonie, NMHDECAY: A Fortran code for the Higgs masses, couplings and decay widths in the NMSSM. JHEP 0502, 066 (2005). arXiv:hep-ph/0406215

33. U. Ellwanger, C. Hugonie, NMHDECAY 2.0: An Updated program for sparticle masses, Higgs masses, couplings and decay widths in the NMSSM. Comput. Phys. Commun. 175, 290-303 (2006). arXiv:hep-ph/0508022

34. G. Belanger, F. Boudjema, C. Hugonie, A. Pukhov, A. Semenov, Relic density of dark matter in the NMSSM. JCAP 0509, 001 (2005). arXiv:hep-ph/0505142

35. U. Ellwanger, C. Hugonie, NMSPEC: A Fortran code for the sparticle and Higgs masses in the NMSSM with GUT scale boundary conditions. Comput. Phys. Commun. 177, 399-407 (2007). arXiv:hep-ph/0612134

36. D. Das, U. Ellwanger, A.M. Teixeira, NMSDECAY: A Fortran Code for Supersymmetric Particle Decays in the Next-to-Minimal Supersymmetric Standard Model. Comput. Phys. Commun. 183, 774-779 (2012). arXiv:1106.5633

37. M. Muhlleitner, A. Djouadi, Y. Mambrini, SDECAY: A Fortran code for the decays of the supersymmetric particles in the MSSM. Comput. Phys. Commun. 168, 46-70 (2005). arXiv:hep-ph/0311167

38. G. Degrassi, P. Slavich, On the radiative corrections to the neutral Higgs boson masses in the NMSSM. Nucl. Phys. B825, 119-150 (2010). arXiv:0907.4682
39. F. Staub, P. Athron, U. Ellwanger, R. Grober, M. Muhlleitner, P. Slavich, A. Voigt, Higgs mass predictions of public NMSSM spectrum generators. 1507, 05093 (2015)

40. G.W. Bennett et al., Final Report of the Muon E821 anomalous magnetic moment measurement at BNL. Phys. Rev. D73, 072003 (2006). arXiv:hep-ex/0602035

41. P.A.R. Ade Planck et al., Results. XVI. Cosmological parameters. Astron. Astrophys. 571(A16), 2014 (2013). arXiv:1303.5076

42. D.S. Akerib et al., First results from the LUX dark matter experiment at the Sanford Underground Research Facility. Phys. Rev. Lett. 112, 091303 (2014). arXiv:1310.8214

43. G. Belanger, B. Dumont, U. Ellwanger, J.F. Gunion, S. Kraml, Global fit to Higgs signal strengths and couplings and implications for extended Higgs sectors. Phys. Rev. D88, 075008 (2013). arXiv: 1306.2941

44. M.Y. Binjonaid, S.F. King, Naturalness of scale-invariant NMSSMs with and without extra matter. Phys. Rev. D 90(5), 055020 (2014). arXiv: 1403.2088

45. L.J. Hall, D. Pinner, J.T. Ruderman, A Natural SUSY Higgs Near 126 GeV. JHEP, 04, 131 (2012). arXiv:1112.2703

46. H. Baer, V. Barger, M. Savoy, Upper bounds on sparticle masses from naturalness or how to disprove weak scale supersymmetry. 1509, 02929 (2015)

47. A. Buckley, PySLHA: a Pythonic interface to SUSY Les Houches Accord data. (2013). arXiv:1305.4194

48. P.Z. Skands et al., SUSY Les Houches accord: Interfacing SUSY spectrum calculators, decay packages, and event generators. JHEP 07, 036 (2004). arXiv:hep-ph/0311123

49. B.C. Allanach et al., SUSY Les Houches Accord 2. Comput. Phys. Commun. 180, 8-25 (2009). arXiv:0801.0045

50. J.F. Gunion, D. Hooper, B. McElrath, Light neutralino dark matter in the NMSSM. Phys. Rev. D 73, 015011 (2006). arXiv:hep-ph/0509024

51. J. Cao, C. Han, L. Wu, P. Wu, J.M. Yang, A light SUSY dark matter after CDMS-II, LUX and LHC Higgs data. JHEP, 1405, 056 (2014). arXiv: 1311.0678

52. T. Han, Z. Liu, S. Su, Light neutralino dark matter: direct/indirect detection and collider searches. JHEP, 1408, 093 (2014). arXiv: 1406.1181

53. U. Ellwanger, A.M. Teixeira, NMSSM with a singlino LSP: possible challenges for searches for supersymmetry at the LHC. JHEP 1410, 113 (2014). arXiv: 1406.7221

54. J.S. Kim, T.S. Ray, The higgsino-singlino world at the large hadron collider. Eur. Phys. J. C75, 40 (2015). arXiv: 1405.3700

55. C. Han, D. Kim, S. Munir, M. Park, $\mathcal{O}(1) \mathrm{GeV}$ dark matter in SUSY and a very light pseudoscalar at the LHC. (2015). arXiv: 1504.05085

56. P. Draper, T. Liu, C.E.M. Wagner, L.T. Wang, H. Zhang, Dark light Higgs. Phys. Rev. Lett. 106, 121805 (2011). arXiv:1009.3963

57. M. Casolino, T. Farooque, A. Juste, T. Liu, M. Spannowsky, Probing a light CP-odd scalar in di-top-associated production at the LHC. (2015). arXiv:1507.07004

58. J. Beuria, A. Chatterjee, A. Datta, S.K. Rai, Two Light Stops in the NMSSM and the LHC. (2015). arXiv:1505.00604

59. A Search for Light CP-Odd Higgs Bosons Decaying to mu+ muin ATLAS. Technical Report ATLAS-CONF-2011-020 (CERN, Geneva, 2011)

60. Search for a light pseudoscalar boson in the dimuon channel. Technical Report CMS-PAS-HIG-12-004 (CERN, Geneva, 2012)

61. G. Aad et al., Search for Higgs bosons decaying to a $8 \mathrm{TeV}$ with the ATLAS experiment. (2015). arXiv:1505.01609

62. V. Khachatryan et al., A search for pair production of new light bosons decaying into muons. (2015). arXiv:1506.00424

63. V. Khachatryan et al., Search for a very light NMSSM Higgs boson produced in decays of the $125 \mathrm{GeV}$ scalar boson and decaying into $\tau 8 \mathrm{TeV}$. (2015). arXiv:1510.06534 
64. V. Khachatryan et al., Searches for electroweak production of charginos, neutralinos, and sleptons decaying to leptons and W, $\mathrm{Z}$, and Higgs bosons in pp collisions at $8 \mathrm{TeV}$. Eur. Phys. J. C 74(9), 3036 (2014). arXiv:1405.7570

65. V. Khachatryan et al., Searches for electroweak neutralino and chargino production in channels with Higgs, Z, and W bosons in pp collisions at 8 TeV. Phys. Rev. D 90(9), 092007 (2014). arXiv: 1409.3168

66. G. Aad et al., Search for the electroweak production of supersymmetric particles in $\sqrt{s}$ collisions with the ATLAS detector. (2015). arXiv: 1509.07152

67. G. Aad et al., ATLAS Run 1 searches for direct pair production of third-generation squarks at the Large Hadron Collider. (2015). arXiv: 1506.08616

68. G. Aad et al., Summary of the searches for squarks and gluinos using $\sqrt{s}=8 \mathrm{TeV}$ pp collisions with the ATLAS experiment at the LHC. (2015). arXiv:1507.05525

69. Commissioning the performance of key observables used in SUSY searches with the first $13 \mathrm{TeV}$ data. (2015)

70. M. Drees, H. Dreiner, D. Schmeier, J. Tattersall, J. Soo Kim, CheckMATE: confronting your favourite new physics model with LHC data. Comput. Phys. Commun. 187, 227-265 (2014). arXiv: 1312.2591

71. J. de Favereau, C. Delaere, P. Demin, A. Giammanco, V. Lemaetre, A. Mertens, M. Selvaggi, DELPHES 3, a modular framework for fast simulation of a generic collider experiment. JHEP 02, 057 (2014). arXiv:1307.6346

72. M. Cacciari, G.P. Salam, G. Soyez, FastJet user manual. Eur. Phys. J. C72, 2012 (1896). arXiv:1111.6097

73. M. Cacciari, G.P. Salam, Dispelling the $N^{3}$ myth for the $k_{t}$ jetfinder. Phys. Lett. B 641, 57-61 (2006). arXiv:hep-ph/0512210

74. M. Cacciari, G.P. Salam, G. Soyez, The Anti-k(t) jet clustering algorithm. JHEP, 04, 063 (2008). arXiv:0802.1189

75. A.L. Read, Presentation of search results: the CL(s) technique. J. Phys. G28, 2693-2704 (2002)
76. T. Sjostrand, S. Mrenna, P.Z. Skands, A brief introduction to PYTHIA 8.1. Comput. Phys. Commun. 178, 852-867 (2008). arXiv:0710.3820

77. T. Sjostrand, S. Mrenna, P.Z. Skands, PYTHIA 6.4 physics and manual. JHEP, 0605, 026 (2006). arXiv:hep-ph/0603175

78. V. Khachatryan et al., Search for physics beyond the standard model in events with two leptons, jets, and missing transverse momentum in pp collisions at sqrt(s) $=8 \mathrm{TeV}$. JHEP, 04, 124 (2015). arXiv:1502.06031

79. Electron performance with $19.6 \mathrm{fb}^{-1} \mathrm{TeV}$ with the CMS detector. (2013)

80. Performance of $\mathrm{b}$ tagging at $\operatorname{sqrt}(\mathrm{s})=8 \mathrm{TeV}$ in multijet, ttbar and boosted topology events. Technical Report CMS-PAS-BTV-13001 (CERN, Geneva, 2013)

81. CMS collaboration, Performance of CMS muon reconstruction in pp collision events at $\sqrt{s}=7 \mathrm{TeV}$. J. Instrum. 7, P10002 (2012). arXiv:1206.4071 (CMS-MUO-10-004. CERN-PH-EP-2012-173. Submitted to the Journal of Instrumentation)

82. M. Kramer, A. Kulesza, R. van der Leeuw, M. Mangano, S. Padhi, T. Plehn, X. Portell, Supersymmetry production cross sections in pp TeV. (2012). arXiv:1206.2892

83. C. Borschensky, M. Kramer, A. Kulesza, M. Mangano, S. Padhi, T. Plehn, X. Portell, Squark and gluino production cross sections in pp collisions at $\sqrt{s}=13,14,33$ and $100 \mathrm{TeV}$. Eur. Phys. J. C 74(12), 3174 (2014). arXiv:1407.5066

84. R. Barate et al., Search for the standard model Higgs boson at LEP. Phys. Lett. B565, 61-75 (2003). arXiv:hep-ex/0306033

85. G. Alexander et al., Search for neutral Higgs bosons in $Z^{0}$ decays using the OPAL detector at LEP. Z. Phys. C 73, 189-199 (1997)

86. T. Barklow, J. Brau, K. Fujii, J. Gao, J. List, N. Walker, K. Yokoya, ILC operating scenarios. 1506, 07830 (2015) 